\title{
Research on Application Mode of VR/AR Technology in Education and Teaching
}

\author{
Wang Yu, Song Chi, Cheng Shi \\ School of Computer Science and Technology, Nantong University, Nantong, Jiangsu, China, 226019
}

Keywords: VR; AR; education and teaching; application mode

Abstract: Virtual Reality (VR) and Augmented Reality (AR) have gradually expanded their application fields due to their unique technical features and have begun to enter the field of education. By summarizing the research basis on the application of augmented reality and virtual reality technology in education, the application modes and technical advantages in education are analyzed, mainly enhancing teaching effect, the participation and creation of dimensions, and collaborative application mode of virtual reality and augmented reality technology in education and teaching is discussed.

\section{Introduction}

With the development of information technology and the popularization of Internet, virtual reality (VR) and augmented reality (AR) are likely to become the next large-scale computing platform, as we saw on PC and smart phones. VR / AR technology may be a standard tool in the field of education and may change the way of education and education. Teachers can use VR / AR as a way for students to interact with objects in the 3D environment. For example, students can understand the solar system or historical events by interacting with these virtual worlds. Therefore, the new technology of VR /AR is bound to bring new educational thinking to solve some difficult problems that could not be solved in the process of education and teaching, and bring a series of great changes to education and teaching.

\section{VR/AR Features}

VR is a comprehensive technology, which combines computer technology, multimedia technology, network technology and simulation technology. It is a computer simulation system that can create and experience the virtual world. It uses computer to generate an interactive 3D dynamic view and entity behavior system with multi-source information fusion [1]. The super high degree of simulation can completely immerse the user into the environment. AR is a technique to calculate the position and angle of the camera image in real time and add the corresponding image.

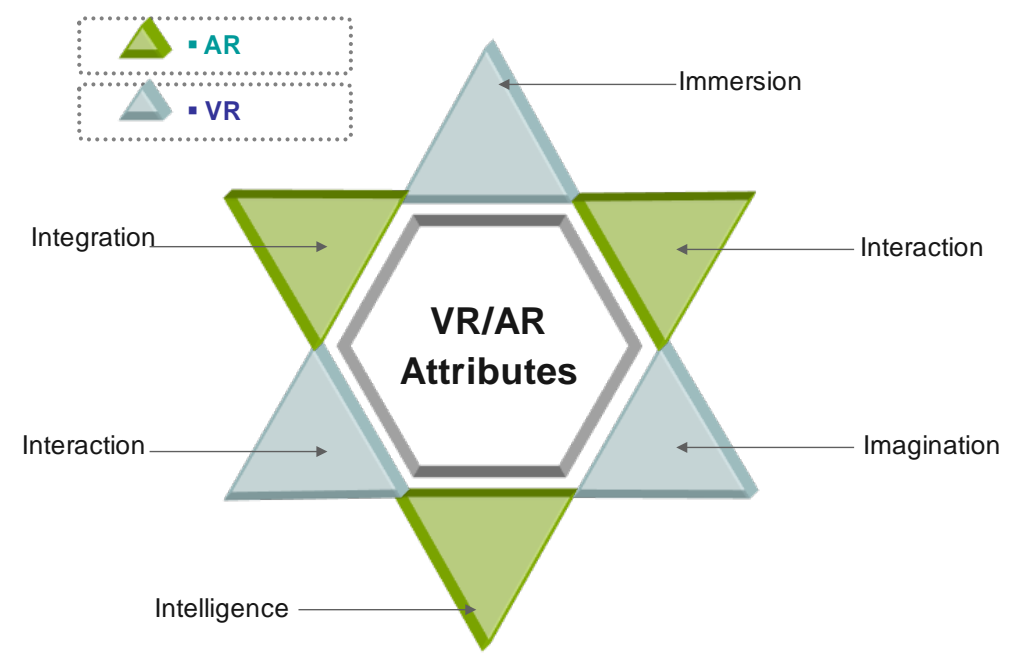

Fig. 1 Attributes of VR and AR 
The goal of this technology is to create and interact with the virtual world in the real world on the screen. This technology not only displays the information of the real world, but also displays the virtual information at the same time. The two kinds of information complement each other, superimpose the virtual world on the real world and interact with each other, breaking through the human senses. The following figure shows the attributes of VR and AR.

\section{Theoretical Basis of VR/AR Application in Education}

When a new technology is applied to the field of education, the key factors include not only the technology itself, but also the examination of the framework of education theory. It requires the support of relevant learning theories and educational theories to guide the practice of education so as to ensure maximize the effectiveness of technical applications, and avoid going astray [2].

\subsection{Immersion theory.}

The immersion theory points out that challenges and skills are the main factors affecting the sense of immersion. If the challenge is too high, users will lack control of the environment, causing anxiety or depression. On the other hand, if the challenge is too low, users will become tired and lose interest. The state of immersion occurs mainly in the balance of the two. Only when high challenges are accompanied by high skills, talent enters and maintains a state of immersion, which they call this state of flow. The immersion experience requires the conditions as shown in Table 1.

Table1. The required conditions for immersion experience

\begin{tabular}{|l|l|}
\hline No. & Conditions \\
\hline 1 & Balance between operator skills and mission challenges \\
\hline 2 & The operator is fully focused \\
\hline 3 & The operator has a clear mission objective \\
\hline 4 & Operators get immediate and immediate feedback continuously \\
\hline 5 & The operator can control the process of the task \\
\hline
\end{tabular}

Virtual Reality and Augmented Reality, with their unique technological advantages, enable learners to throw away the interference of the surrounding tools and concentrate their attention on learning contents in the learning process, so as to enter the immersion state [3]. The complex relationship between the theory of reveal immersed challenge skills and the general conditions required to achieve the flow state, provides a feasible theoretical guidance to the research based on VR/AR technology learning environment, and on how to properly and reasonably use the relationship, create conditions so as to make the learners immerse, immersed into the learning state.

\subsection{Situational cognitive learning theory.}

Situational cognitive learning theory holds that real activities and scenarios are the source of knowledge, context is the basis of all cognitive activities, and the nature of cognitive processes is determined by context [3]. Only in the application process can we fully understand the knowledge. Therefore, if people really understand the meaning of knowledge and use knowledge flexibly, they must be in a real and rich social scene. Understand in real situations. Real activities should become the center of learning. Knowledge and action are interactive, knowledge is contextual, knowledge is part of activities, backgrounds, culture and they are constantly applied and developed in rich contexts through activities, and continuous applied and developed through the activities in the rich context. Situational learning emphasizes learning knowledge and mastering skills in context, content, background, community of practice, and participation as the four basic elements of situational learning.

VR/AR technology has a natural fit with situational learning. Through VR/AR technology, it is very easy to create learning situations. Students can actually experience the inner relationship between learning content and real life in the process of learning. Moving from one situation to another, that is, migratory ability is a very important skill. Enhancement can actually improve the ability to 
migrate.

\section{Application Mode of VR/AR in Education \& Teaching}

The development of information technology has brought a revolutionary impact on education and teaching, and the in-depth application of information technology will certainly lead to the comprehensive reform of educational goals, content, models, and methods. As an emerging technology, VR/AR has become increasingly prominent in education and teaching. Applying VR/AR technology to education will inevitably inject new vigor and vitality into education. On the one hand, VR/AR technology will be applied. Daily teaching will bring about changes in the field of teaching and enhance the effectiveness of teaching. On the other hand, the use of VR/AR technology can expand the learning space and enrich the forms and experiences of learning.

\subsection{Improve the learning environment.}

In addition to the functions of perception, recognition, support, and feedback, the learning environment should also be contextual. Because, only in the context of effective learning can really happen. According to the mode of situational learning, knowledge must be learned in context. The situation can be a real work scenario or a virtual substitute for the real work environment. However, providing real learning situations is easily impeded by the constraints of actual conditions. The advent of VR/AR technology has raised the realism of the simulation environment to an unprecedented level, allowing learners to interact in real time and carry out task collaboration. VR/AR technology provides richer support for situational awareness and learning. With augmented reality technology, it is easy to create realistic learning situations and allow learners to immerse themselves in learning activities, thereby improving the efficiency of learning.

\subsection{Innovate the teaching mode.}

In the traditional teaching mode, the teaching activities include teachers, students, and teaching materials. In this mode, teachers are the main body of teaching, and students passively accept knowledge. However, in the teaching mode supported by VR/AR technology, the relationship among teachers, students, teaching materials, and media is student-centered and teacher-oriented [4]. The learning environment created with VR/AR gives full play to the enthusiasm and initiative of students and ultimately enables students to master the content of learning.

VR/AR technology supports situational learning and in-depth interaction during the learning process, which helps students acquire and construct their knowledge and experience [5]. Under this structure of activities, educators can stimulate the learners' senses through the presentation of rich media information and inspire learners' motivation, they can gradually guide the learners' learning by setting up multiple tasks; through cooperation and communication, they can feedback and other links to ensure that students' learning is conducted efficiently.

\subsection{Expand learning space and styles.}

There are more and more opportunities and ways for students to learn independently. The proportion of non-formal learning is increasing day by day. Education must face the increasing integration of formal and informal learning. In fact, compared to formal school teaching, the application of VR/AR technology in the informal learning field has broader prospects, such as augmented reality e-books, mobile learning based on augmented reality, and augmented reality educational games.

\section{Summary}

This article details the current status of the application of VR/AR technology in education and education, analyzes the educational application value and technical advantages of VR/AR, and briefly describes the theoretical basis of VR/AR technology in education applications, and discusses the application of VR/AR technology in education from two dimensions, which is strengthens the 
teaching effect and expands the learning space. With the development of science and technology, VR/AR technology also rapidly penetrates into different fields. From the perspective of education, thinking about the combination of VR/AR technology and education and teaching, and the revolution of VR/AR technology in education and teaching will not only help to guide the educational practice, so as to ensure the maximization of technical application effects, it also lays a good foundation for further detailed application of new technologies.

\section{Acknowledgement}

2017 Research Project on Modern Educational Technology in Jiangsu Province (No.2017-R-54131)

\section{References}

[1] Shelton Hedley. Using Augmented Reality for Teaching Earth-Sun Relationships to Undergraduate Geography Students [C]. Augmented Reality Toolkit. The First IEEE International Workshop, 2012, p.30

[2] Kaufmann. The potential of augmented reality in dynamic geometry education[C]. 12th International Conference on Geometry and Graphics, Salvador, Brazil. 2006, p.13

[3] Chih-Hsiang Ko. The Application of Augmented Reality to Design Education[C]. Edutainment, 2011, p.54

[4] Jong Weon Lee. Mathematical Education Game Based on Augmented Reality[A]. Proceeding of Edutainment [C]. Berlin: Springer-Verlag, 2008, p.450

[5] Zhang Hongbo. Research on Application of Augmented Reality Technology in Teaching [D]. Kaifeng: Henan University, 2012, p.27 